\title{
Protein tyrosine phosphatase 1B inhibitors isolated from Artemisia roxburghiana
}

\author{
Muhammad Raza Shah ${ }^{1}$, Ishtiaq ${ }^{2}$, Syed Muhammad Hizbullah ${ }^{3}$, Solomon Habtemariam ${ }^{4}$, Armando Zarrelli ${ }^{5}$, Akhtar $^{2}$ \\ Muhammad $^{6}$, Simona Collina ${ }^{7}$, and Inamulllah Khan ${ }^{3}$ \\ ${ }^{1}$ H.E.J. Research Institute of Chemistry, International Center for Chemical and Biological Sciences, University of Karachi, Karachi, Pakistan, \\ ${ }^{2}$ Department of Chemistry, Hazara University, Mansehra, Pakistan, ${ }^{3}$ Department of Pharmacy, University of Peshawar, Peshawar, Pakistan, \\ ${ }^{4}$ Pharmacognosy Research Laboratories, Medway School of Science, University of Greenwich, Kent, UK, ${ }^{5}$ Department of Chemical Sciences, \\ University of Naples "Federico II", Naples, Italy, ${ }^{6}$ Department of Chemistry, University of Karachi, Karachi, Pakistan, and ${ }^{7}$ Department of Pharmacy, \\ University of Pavia, Pavia, Italy
}

\section{Abstract}

Artemisia roxburghiana is used in traditional medicine for treating various diseases including diabetes. The present study was designed to evaluate the antidiabetic potential of active constituents by using protein tyrosine phosphatase 1B (PTP1B) as a validated target for management of diabetes. Various compounds were isolated as active principles from the crude methanolic extract of aerial parts of $A$. roxburghiana. All compounds were screened for PTP1B inhibitory activity. Molecular docking simulations were performed to investigate the mechanism behind PTP1B inhibition of the isolated compound and positive control, ursolic acid. Betulinic acid, betulin and taraxeryl acetate were the active PTP1B principles with $\mathrm{IC}_{50}$ values $3.49 \pm 0.02,4.17 \pm 0.03$ and $87.52 \pm 0.03 \mu \mathrm{M}$, respectively. Molecular docking studies showed significant molecular interactions of the triterpene inhibitors with Gly220, Cys215, Gly218 and Asp48 inside the active site of PTP1B. The antidiabetic activity of $A$. roxburghiana could be attributed due to PTP1B inhibition by its triterpene constituents, betulin, betulinic acid and taraxeryl acetate. Computational insights of this study revealed that the $\mathrm{C}-3$ and $\mathrm{C}-17$ positions of the compounds needs extensive optimization for the development of new lead compounds.
\end{abstract}

\section{Keywords}

Artemisia roxburghiana, docking, PTP1B, triterpenes, ursolic acid

\section{Introduction}

Protein tyrosine phosphatases (PTPs) are enzymes that play pivotal role in cellular processes, such as cell growth, proliferation and differentiation. Several recent studies further revealed that PTPs are involved in various metabolic activities, immunological responses, cell-cell adhesions and cell-matrix interactions ${ }^{1-3}$. Protein tyrosine phosphatase 1B (PTP1B), is an intracellular nonreceptor type PTP, which is considered to be a well-validated therapeutic target for many diseases including diabetes. Extensive biochemical and genetic investigations have shown that PTP1B is involved in the negative control of insulin- and leptin-receptor ${ }^{4-6}$. Other evidence from PTP1B-knockout mice further revealed an increased sensitivity to insulin, enhanced glycemic control and resistant to diet-induced obesity ${ }^{7}$. Hence, inhibition of PTP1B offers a novel approach in regulating type-2 diabetes and obesity. Accordingly, researches in many laboratories worldwide are currently focusing in the search of novel PTP1B inhibitors from synthesis and natural sources.

Artemisia roxburghiana Besser is a perennial aromatic herb belonging to the family Asteraceae. In Pakistan, where the plant is native and occupying dry open slopes and waste lands of the

Address for correspondence: Dr Inamullah Khan, $\mathrm{PhD}$, Department of Pharmacy, University of Peshawar, Peshawar, KPK, Pakistan. Tel: +929216750. Fax: +92-9216750. E-mail: inam_marwat333@yahoo.com; inamullah@upesh.edu.pk
Himalayan region, various ethnobotanical uses have been reported. These include for treating fever, rheumatism, malaria, dysentery and hepatitis ${ }^{8,9}$. The aerial parts are also used to treat diabetes either alone or in combination with other plants, such as the dried fruits of Zizyphus jujube 9 . In support of this latter traditional use, the ethanolic extract of $A$. roxburghiana from Pakistan has previously shown to display insulin secretagogue activity in cultured insulinoma cell line, INS- 1 cells ${ }^{10}$. Apart from preliminary phytochemical studies, such as essential oils analysis by $\mathrm{GC}-\mathrm{MS}^{9-11}$, the active antidiabetic principles of the plants have not yet been investigated. Owing to the prominent role of PTP1B in diabetes, we herewith identified the active components of $A$. roxburghiana using this pharmacological target. Through molecular docking simulation studies, we have also shown, for the first time, the molecular mechanism of PTP1B inhibition by the isolated compounds and the standard positive control, ursolic acid.

\section{Experimental}

\section{General phytochemical analysis methodology}

${ }^{1} \mathrm{H}$ NMR, ${ }^{13} \mathrm{C}$ NMR and 2D-NMR (COSY, NOESY, HMQC and HMBC experiments) spectra were obtained on a Bruker $600 \mathrm{MHz}$ instrument (Billerica, MA). Homonuclear ${ }^{1} \mathrm{H}$ connectivities were determined by using the COSY experiment. One bond ${ }^{1} \mathrm{H}-{ }^{13} \mathrm{C}$ connectivities were determined with HMQC while two- and threebond ${ }^{1} \mathrm{H}-{ }^{13} \mathrm{C}$ connectivities were determined by $\mathrm{HMBC}$ 
experiments, as reported earlier. Chemical shifts were reported in $\delta$ (ppm) using the solvent $\left(\mathrm{CD}_{3} \mathrm{OD}\right)$ standard and coupling constants $(J)$ were measured in Hz. A Jeol JMS HX 110 mass spectrometer (Tokyo, Japan) using glycerol as the matrix was used for mass spectrometry. HREI MS was carried out on Jeol JMS 600 mass spectrometer (Tokyo, Japan). Silica gel 60 (Merck, Darmstadt, Germany) was used as an adsorbent for open column chromatography. TLC analysis was routinely performed using hexane:EtOAc mixtures as the mobile phase and compounds were visualized under UV light.

\section{Plant material}

The aerial parts (green stems with leaves) of A. roxburghiana were collected from the University campus of Hazara University Mansehra, Pakistan. The authenticated voucher specimen (no. 3486) was then deposited at the Herbarium of Hazara University, Mansehra, Pakistan. The plant materials were shade dried and ground into powder.

\section{Extraction and isolation}

The powdered plant materials $(17 \mathrm{~kg})$ were extracted three times by soaking with $24 \mathrm{~L}$ of methanol for two weeks. Combined extracts were filtered and evaporated under reduced pressure to yield $1.5 \mathrm{~kg}$ of the crude extract. Preliminary fractionation of the crude extract was done by suspending the crude extract in water and extraction using solvents of increasing polarity:hexane (4.2L), chloroform (7.8 L), ethyl acetate $(6 \mathrm{~L})$ and n-butanol (3L). Remaining unfractionated crude extract was taken as aqueous fraction. The ethyl acetate fraction $(160 \mathrm{~g})$ that showed the highest PTP1B inhibitory activity was subjected to silica gel column chromatography $(\mathrm{CC}, 700 \mathrm{~mm} \times 80 \mathrm{~mm})$ with hexane containing increasing percentage of ethyl acetate used as eluents. Fractions obtained from the 4.0:6.0 to 0:10 hexane:EtOAc mixtures were further subjected to $\mathrm{CC}$ under the same conditions to yield six compounds: apigenin-7,4-dimethyl ether $(\mathbf{1}, 48.4 \mathrm{mg}$, $\mathrm{R}_{\mathrm{F}} 0.56$ in hexane:ethyl acetate $\left.8.5: 1.5\right)$; taraxeryl acetate (2, $271 \mathrm{mg}, \mathrm{R}_{\mathrm{F}} 0.75$ in hexane:ethyl acetate $\left.0.5: 9.5\right)$; $9 \mathrm{mg}$ of a compound which was identified as betulin $\left(3,94 \mathrm{mg}, \mathrm{R}_{\mathrm{F}} 0.72\right.$ in hexane:ethyl acetate $0.8: 9.2)$; betulinic acid $\left(4,9.3 \mathrm{mg}, \mathrm{R}_{\mathrm{F}} 0.75\right.$ in hexane:ethyl acetate 0.8:9.2); 7-Hydroxy-6-methoxy-coumarin (Scopoletin, 5, $42 \mathrm{mg}, \mathrm{R}_{\mathrm{F}} 0.5$ in hexane:ethyl acetate 1:9), and 6,7-dimethoxy-coumarin $\left(6,5 \mathrm{mg}, \mathrm{R}_{\mathrm{F}} 0.57\right.$ in hexane:ethyl acetate 1:9). The structures of all the isolated compounds were confirmed by comparing their corresponding NMR and mass spectral data with those previously reported ${ }^{12-17}$.

\section{PTP1B inhibitory activity}

The enzyme inhibition assay was carried out in 96-well plates using 3,3-dimethyl glutarate buffer ( $\mathrm{pH} 7.0)$. The reaction mixture contained various concentrations of test drugs, $p$-nitrophenol phosphate $(p N P P, 1 \mathrm{mM})$ and PTB1B $(10 \mathrm{mM})$. After incubation at $27{ }^{\circ} \mathrm{C}$ for $40 \mathrm{~min}$, the absorbance of the released $p$ NPP was measured at $405 \mathrm{~nm}$. All assays were carried out in triplicates from which $\mathrm{IC}_{50}$ values were calculated.

\section{Molecular docking simulations}

OEDocking 3.0.0 (Santa Fe, NM) ${ }^{18-22}$ was used in this study to dock the OMEGA pre-generated multi-conformer library. FRED strategy was used, which exhaustively dock/score all possible positions of each ligand in the binding site. The exhaustive search is based on rigid rotations and translations of each conformer within the binding site defined by a box. FRED filtered the poses ensemble by rejecting the ones that clash with the protein (PTP1B) or that does not have enough contacts with the protein.
The final poses can then be scored or re-scored using one or more scoring functions. In this study, the smooth shape-based Gaussian scoring function (shapegauss) was selected to evaluate the shape complementarily between each ligand and the binding pocket. Default FRED protocol was used except for the size of the box defining the binding sites. In an attempt to optimize the docking-scoring performance, we performed exhaustive docking with shapegauss applying the "Optimization" mode. The "Optimization" mode involves a systematic solid body optimization of the top ranked poses from the exhaustive docking. Three different boxes were explored for PTP1B (PDB:2BGD). Three different simulations were carried out with an added value of $8 \AA$ around the reference ligand. This method was validated using the comparison of docked pose of the reference compound in PDB file versus its crystal structure. RMSD values was found to be 0.466 , which seems to quite satisfactory.

\section{Results and discussion}

Apart from some preliminary studies showing the in vitro insulin secretagogue activity $A$. roxburghiana ${ }^{10}$, scientific data to justify the antidiabetic traditional uses of the plant is not available. In the present study, PTP1B inhibitory activity was used as a validated target for sourcing potentially useful antidiabetic compounds from the plant. In this in vitro assay, the crude extract showed considerable $(59.2 \pm 0.11 \%)$ enzyme inhibitory activity at the concentration of $100 \mu \mathrm{g} / \mathrm{mL}$. In order to isolate the active PTP1B inhibitory principles of the plant, the crude extract was fractionated with solvents of increasing polarity. When screened at the fixed concentration of $100 \mu \mathrm{g} / \mathrm{mL}$, the most active fraction was ethyl acetate that displayed $68.2 \pm 0.13 \%$ activity followed by the crude extract $(52.6 \pm 0.18 \%)$, chloroform $(46.3 \pm 0.14 \%)$, aqueous $(42.1 \pm 0.09), n$-butanol $(39.4 \pm 0.12)$ and $n$-hexane $(21.7 \pm 0.11 \%)$. Repetitive column chromatography over silica gel of the most active ethyl acetate fraction afforded six compounds, which were identified as apigenin-7,4-dimethyl ether (1); taraxeryl acetate (2); betulin (3); betulinic acid (4); 7-hydroxy-6-methoxy-coumarin (Scopoletin, 5) and 6,7dimethoxy-coumarin (6, Figure 1). The spectral data of these previously known compounds from other sources were in good agreement with those published before by various authors ${ }^{12-17}$. All of the isolated compounds together with the positive control, ursolic acid, were subjected to PTP1B inhibition assay. The relative activity of these compounds is shown in Table 1.

Ursolic acid is a pentacyclic triterpenoid acid that is commonly used as a standard PTP1B inhibitory agent. The activity of this standard positive control recorded in the present study (Table 1) was in good agreement with those reported for the compound previously ${ }^{17,23}$. Betulinic acid is another pentacyclic triterpene in which hydroxyl and carboxyl groups located at similar positions but differ from ursolic acid in one of the ring system (5-membered ring in betulinic acid, Figure 1). Hence, betulinic acid, as with previous reports ${ }^{24}$, displayed potent PTP1B inhibitory activity (Table 1). The considerable role of the carboxylic acid moiety of betulinic acid was demonstrated as its replacement by hydroxyl moiety as in $\mathbf{3}$ (betulin) resulted in a slight decrease in activity by approximately $25 \%$. Similarly, compound 2 (taraxeryl acetate), which lacks both the hydroxyl and carboxylic acid moieties was over many fold less active than betulinic acid (4, Table 1). In comparison to the triterpene compounds, the flavonoid (1) and coumarins $(\mathbf{5}, \mathbf{6})$ derivatives isolated from the active ethyl acetate fraction did not show any activity. Since, some of the compounds identified in the present study were known to occur in A. roxburghiana ${ }^{25}$, our study appears to be the first report on the identification of active compounds against PTP1B principles of the plant. The antidiabetic effect of betulinic acid could also be 
Figure 1. Chemical structures of the isolated compounds (1-6) and ursolic acid (7).<smiles>COc1ccc(-c2cc(=O)c3c(O)cc(OC)cc3o2)cc1</smiles>

(1)<smiles>C=C(C)[C@H]1CC[C@]2(CO)CC[C@]3(C)C(CCC4[C@@]5(C)CC[C@@H](O)C(C)(C)C5CC[C@]43C)C12</smiles>

(3)<smiles>COc1cc2c(=O)occc2cc1O</smiles>

(5)

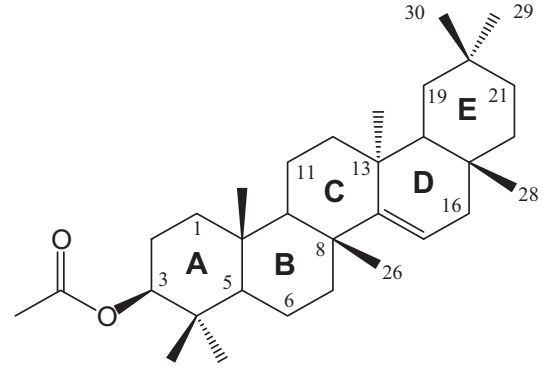

(2)<smiles>C=C(C)[C@H]1CC[C@]2(C(=O)O)CC[C@]3(C)C(CCC4[C@@]5(C)CC[C@@H](O)C(C)(C)C5CC[C@]43C)C12</smiles>

(4)<smiles>COc1cc2ccoc(=O)c2cc1OC</smiles>

(6)<smiles>C[C@H]1CC[C@]2(C(=O)O)CC[C@]3(C)C(=CCC4[C@@]3(C)CC[C@]3(C)C(C)(C)[C@@H](O)CC[C@]43C)[C@@]12C</smiles>

(7)

Table 1. PTP1B activity of A. roxburghiana compounds and ursolic acid*.

\section{Compound}

1. Apigenin-7,4-dimethyl ether

2. Taraxeryl acetate

3. Betulin

4. Betulinic acid

5. Scopoletin

6. 6,7-Dimethoxy-coumarin

7. Ursolic acid (reference)

NA: Not active up to the concentration of $100 \mu \mathrm{M}$ (give the highest tested).

*Data are mean and SEM values from three separate experiments.

mediated through other effects including inhibition of pancreatic lipase and adipocyte lipolysis ${ }^{26}$ and also by enhancing glucose uptake in adipocytes after long treatment ${ }^{27}$. The PTP1B inhibitory effect of betulin has not been reported before but it is known to improve hyperlipidemia and insulin resistance in experimental animals $^{28}$.

Ursolic acid and its derivatives are reported with significant PTP1B inhibitory activities. Through this mechanism, they are known to enhance insulin receptor phosphorylation and stimulate glucose uptake ${ }^{23}$. Mechanism and molecular modeling studies of ursolic acid against PTP1B enzyme has been reported ${ }^{29}$. The mechanism of interaction of these compounds (other than ursolic acid) with PTP1B, however, has not yet been established. In the present study, molecular docking analysis was employed to reveal the favorable binding interactions between the enzyme and triterpene inhibitors. Hydrogen bonding, dipole-dipole attractive forces, hydrophobic interactions and certain other molecular interactions are commonly found and investigated in ligandprotein complexes, which contributes to strengthening, such as macromolecular interactions ${ }^{29-34}$. The secondary hydroxyl group of betulin (17-OH) showed significant and multiple bonding interactions with important amino acid residues of PTP1B (Figures 2 and 3). Interestingly, the amino and secondary amide groups of Gly218, were found to be held by two hydrogen 


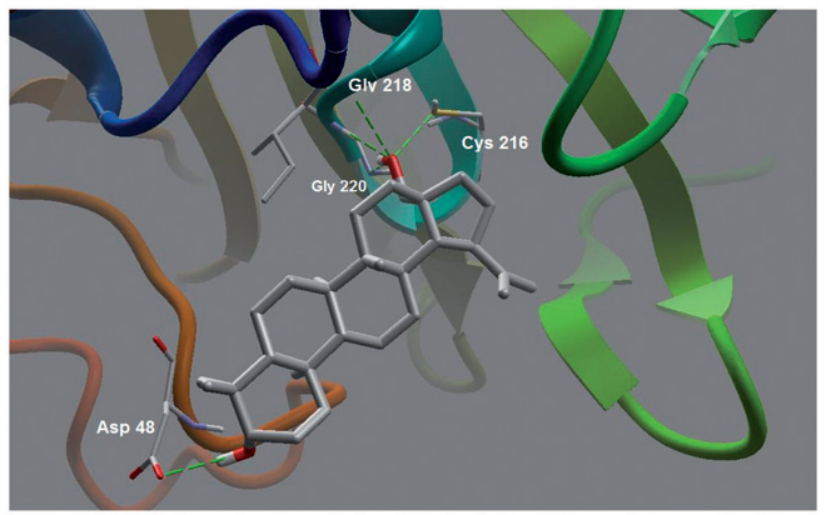

Figure 2. Molecular binding mode of betulin inside catalytic site of PTP13. Hydrogen atoms (except polar ones) were omitted for clarity.

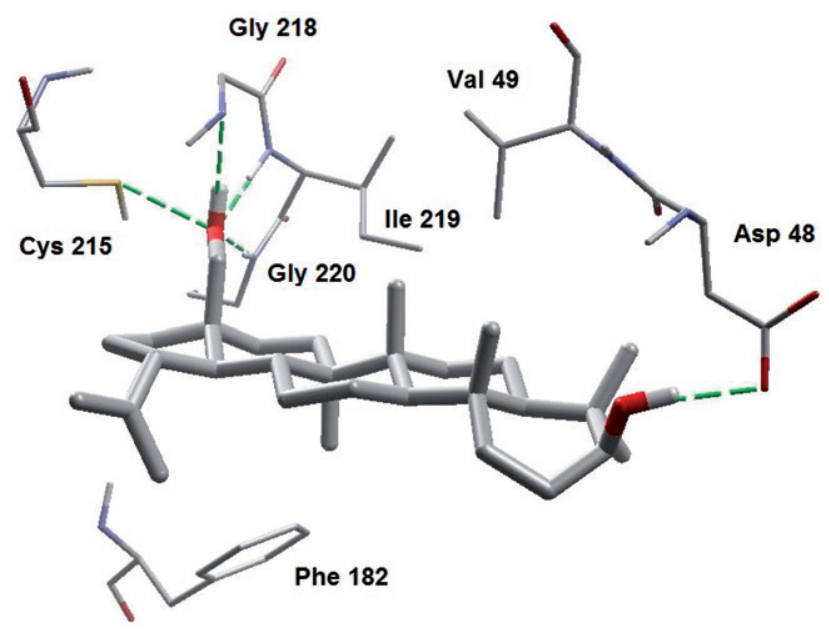

Figure 3. A closer view of betulin bound to the active site of PTP13.

bonding interactions of the 17-OH group, at a distance of 2.99 and $2.76 \AA$, respectively. Similarly, Gly 220 and Cys 215 were found to interact through hydrogen bonding at a distance of 2.97 and $2.72 \AA$, respectively. The other hydroxyl group at C-3 position also played a critical role in protein-ligand complex through hydrogen bonding with Asp 48 (2.76 $\AA$ ): a terminal amino acid residue lining the outer rim of active site of PTP1B. Apart from polar interactions, favorable hydrophobic interactions were detected between Val49 and the two methyl groups of the triterpene skeleton at C-8 and C-10 positions. On the opposite side, Phe182 was favorably supported by interaction with the methyl group at C-14 position. Ile219 also exhibited hydrophobic interactions with cyclic carbons at $\mathrm{C}-15$ and $\mathrm{C}-16$ positions. Molecular shape and electrostatic behavior (Figure 4) of betulin seems to be a major factor responsible for its potent inhibitory activity. Molecular binding modes and interactions of betulinic acid was almost the same as that of betulin (Figure 5). Carboxylic acid group produced slight increase in inhibitory effect of betulinic acid in comparison to betulin.

Most of the molecular interactions for ursolic acid were also similar with betulin and betulinic acid. Few additional hydrogen bonding interactions were, however, found of which the carboxylic acid moiety played a critical role. The conformation of ring $\mathrm{E}$ of ursolic acid made it favorable for the carboxylic acid group to interact with $\operatorname{Arg} 221(3.021 \AA)$ and Ser $216(2.753 \AA)$ via hydrogen bonding (Figures 6 and 7). This finding was in agreement with the finding of taraxeryl acetate that lacks a carboxylic acid moiety, with considerably weaker PTP1B

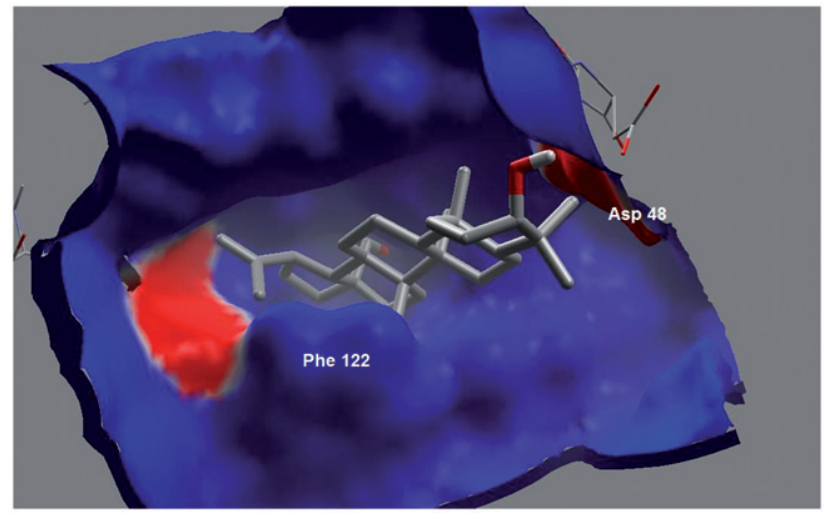

Figure 4. Electrostatic surface of PTP1B. Blue color indicates positive electrostatic surface, red color region indicates negative electrostatic surface and white region depicts non-polar (hydrophobic) surface of the enzyme.

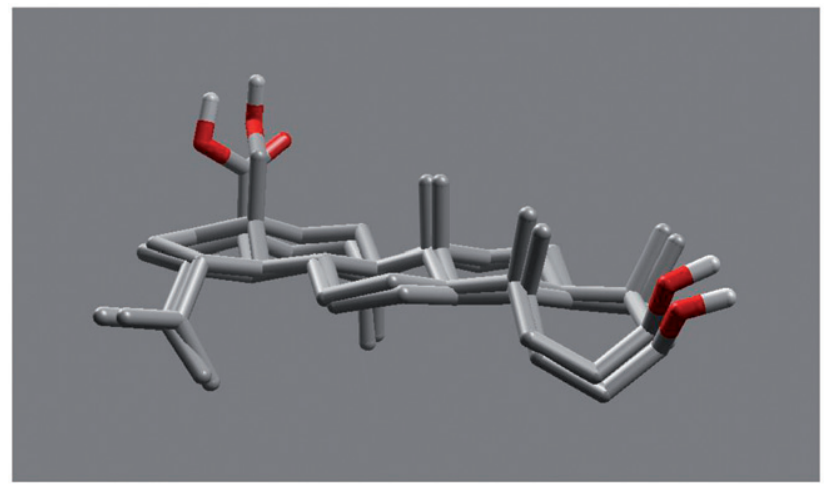

Figure 5. Similar binding modes of betulin and betulic acid. Both poses of the compounds seems to be superimposed on each other. It clearly depicts similar bonding interactions between the enzyme and inhibitors.

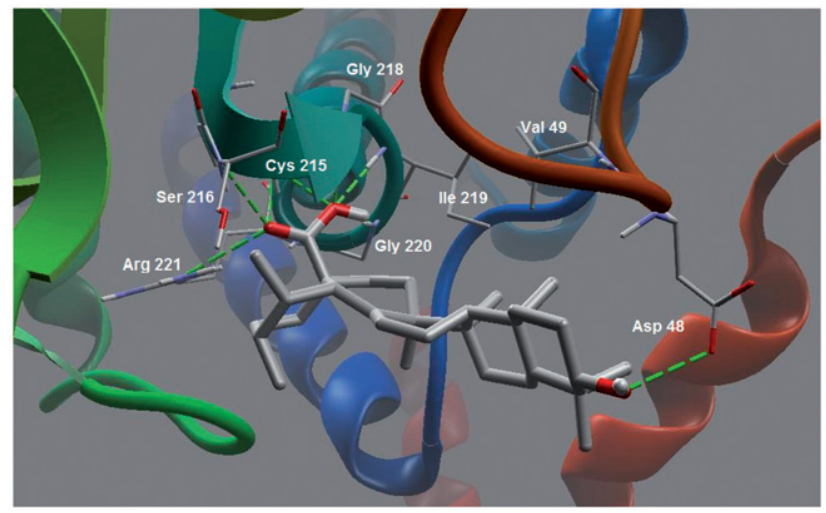

Figure 6. Molecular binding mode of ursolic acid inside active site of PTP13.

inhibitory activity (Table 1). The lack of C-3 hydroxyl group in taraxeryl acetate that would have engaged in hydrogen bonding interaction with Asp48 is a further reason for its weaker PTP1B inhibitory activity.

The overall antidiabetic effect of A. roxburghiana could be attributed to multiple effects including the present finding of PTP1B inhibition, insulin secretagogue activity ${ }^{10}$ and other effects reported for the active ingredients, betulin and betulinic $\operatorname{acid}^{25-27}$. Experimental data obtained from our computational insights study further revealed that the C-3 hydroxyl and C-17 


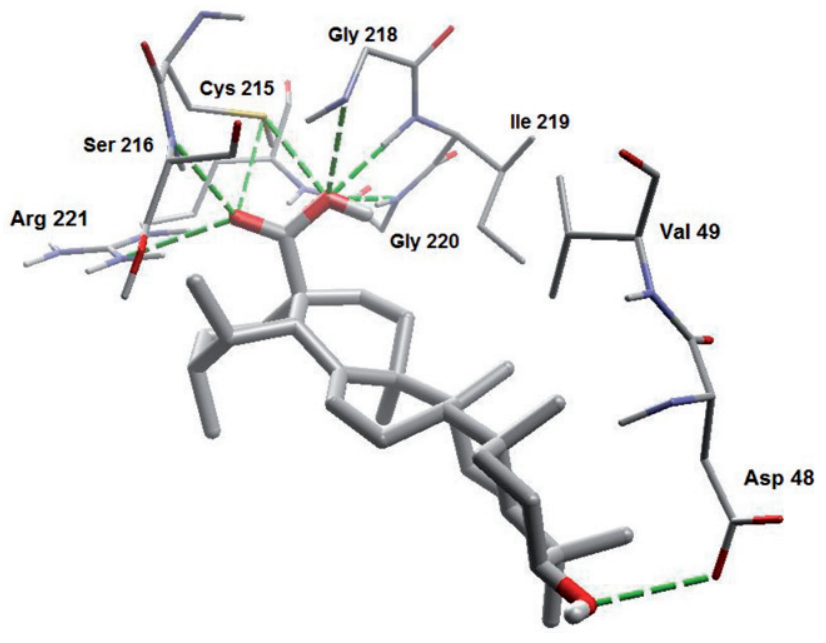

Figure 7. A closer view of ursolic acid bound to the active site of PTP1B.

carboxylic acid positions of the pentacyclic triterpene skeleton are critical for designing new and better PTP1B inhibitors.

\section{Declaration of interest}

The authors declare no conflict of interest.

\section{References}

1. Tonks NK. Protein tyrosine phosphatases: from genes, to function, to disease. Nat Rev Mol Cell Biol 2006;7:833-46.

2. Fisher EH, Charbonneau H, Tonks NK. Protein tyrosine phosphatases: a diverse family of intracellular and transmembrane enzymes. Science 1991;253:401-6.

3. Hunter T. Protein kinases and phosphatases: the Yin and Yang of protein phosphorylation and signaling. Cell 1995;80:225-36.

4. Bialy L, Waldmann $\mathrm{H}$. Inhibitors of protein tyrosine phosphatases: next-generation drugs? Angew Chem 2005;44:3814-39.

5. Koren S, Fantus IG. Inhibition of the protein tyrosine phosphatase PTP1B: potential therapy for obesity, insulin resistance and type-2 diabetes mellitus. Best Pract Res Clin Endocrinol Metabol 2007;21: 621-40.

6. Elchebly M, Payette P, Michaliszyn E, Cromlish W. Increased insulin sensitivity and obesity resistance in mice lacking the protein tyrosine phosphatase-1B gene. Science 1999;283:1544-8.

7. Ali MI, Ketsawatsomkron P, Chantemele EJ, et al. Deletion of protein tyrosine phosphatase $1 \mathrm{~b}$ improves peripheral insulin resistance and vascular function in obese, leptin-resistant mice via reduced oxidant tone. Circ Res 2009;105:1013-22.

8. Dua VK, Verma G, Agarwal DD, et al. Antiprotozoal activities of traditional medicinal plants from the Garhwal region of North West Himalaya, India. J Ethnopharmacol 2011;136:123-8.

9. Ashraf M, Hayat MQ, Jabeen S, et al. Artemisia L. species recognized by the local community of northern areas of Pakistan as folk therapeutic plants. J Med Plant Res 2010;4:112-19.

10. Hussain Z, Waheed A, Qureshi RA, et al. The effect of medicinal plants of Islamabad and Murree region of Pakistan on insulin secretion from INS-1 cells. Phytother Res 2004;18:73-7.

11. Bicchi C, Rubiolo P, Marschall H, et al. Constituents of Artemisia roxburghiana Besser essential oil. Flavour Fragrance J 1998;13: 40-6.

12. Gohari AR, Ebrahimi H, Saeidnia S, et al. Flavones and flavone glycosides from Salvia macrosiphon Boiss. Iranian J Pharma Res 2011;10:247-51.
13. Ogihara K, Higa M, Hokama K, Suga T. Triterpenes from the leaves of Parsonsia laevigata. Phytochemistry 1987;26:783-5.

14. Sharma P, Gupta YK, Sharma MC, Dobhal MP.Two new compounds from the stem of Nerium oleander. Indian J Chem Sect B Org Chem Med Chem 2010;49B:374-8.

15. Ayatollahi AM, Ghanadian M, Afsharypour S, et al. Pentacyclic triterpenes in Euphorbia microsciadia with their T-cell proliferation activity. Iranian J Pharma Res 2011;10:287-94.

16. Ting Z, Yan Y, Guanhua D, Ruoyun C. Study on chemical constituents from the roots of Saussurea lappa. Zhongguo Zhongyao Zazhi 2011;36:1620-2.

17. Chen JX, Huang SH, Wang Y, et al. Studies on the chemical constituents from Lobelia chinensis. J Chinese Med Mat 2010;33 1721-4.

18. Khan I, Nisar M, Shah MR, et al. Anti-inflammatory activities of Taxusabietane A isolated from Taxus wallichiana Zucc. Fitoterapea 2011;82:1003-7.

19. Rizvi SUF, Siddiqui HL, Nisar M, et al. Discovery and molecular docking of quinolyl-thienyl chalcones as anti-angiogenic agents targeting VEGFR-2 tyrosine kinase. Bioorg Med Chem Lett 2012; 22:942-4.

20. Khan I, Nisar M, Khan N, et al. Structural insights to investigate Conypododiol as a dual cholinesterase inhibitor from Asparagus adscendens. Fitoterapia 2010;81:1020-5.

21. Nisar M, Kaleem WA, Khan I, et al. Molecular simulations probing Kushecarpin A as a new lipoxygenase inhibitor. Fitoterapia 2011;82: 1008-11.

22. Khan I, Samad A, Khan AZ, et al. Molecular interactions of 4-acetoxy-plakinamine B with peripheral anionic and other catalytic subsites of the aromatic gorge of acetylcholinesterase: computational and structural insights. Pharma Biol 2013;51:722-7.

23. Zhang W, Hong D, Zhou Y, et al. Ursolic acid and its derivative inhibit protein tyrosine phosphatase $1 \mathrm{~B}$, enhancing insulin receptor phosphorylation and stimulating glucose uptake. Biochim Biophys Acta 2006;1760:1505-12.

24. Choi JY, Na MK, Hwang IH, et al. Isolation of betulinic acid, its methyl ester and guaiane sesquiterpenoids with protein tyrosine phosphatase 1B inhibitory activity from the roots of Saussurea lappa C.B.Clarke. Molecules 2009;14:266-72.

25. Shah MR, Shamim A, White LS, et al. The anti-inflammatory properties of Au-scopoletin nanoconjugates. New J Chem 2014;38: 5566-72.

26. Kim J, Lee YS, Kim C-S, Kim JS. Betulinic acid has an inhibitory effect on pancreatic lipase and induces adipocyte lipolysis. Phytother Res 2012;26:1003-6.

27. Castellano JM, Guinda A, Delgado T, et al. Biochemical basis of the antidiabetic activity of oleanolic acid and related pentacyclic triterpenes. Diabetes 2013;62:1791-9.

28. Tang JJ, Li JG, Qi W, et al. Inhibition of SREBP by a small molecule, betulin, improves hyperlipidemia and insulin resistance and reduces atherosclerotic plaques. Cell Metab 2011;13:44-56.

29. Ramírez-Espinosa JJ, Rios MY, López-Martínez S, et al. Antidiabetic activity of some pentacyclic acid triterpenoids, role of PTP-1B: in vitro, in silico, and in vivo approaches. Eur J Med Chem 2011;46:2243-51.

30. Fazal-Ur-Rehman, Khan MF, Khan I, et al. Molecular interactions of an alkaloid euchrestifoline as a new acetylcholinesterase inhibitor. Bangladesh J Pharmacol 2013;8:361-4.

31. Rehman UU, Shah J, Khan MA, et al. Molecular docking of taraxerol acetate as a new COX inhibitor. Bangladesh J Pharmacol 2013;8:194-7.

32. Ali S, Nisar M, Iriti M, et al. Biotransformation of finasteride by Ocimum sanctum L., and tyrosinase inhibitory activity of transformed: experimental and computational insights. Steroids 2014;92: $20-4$.

33. Khan I, Nisar M, Khan AZ, et al. Molecular insights to explore abietane diterpenes as new LOX inhibitors. Chem Res 2013;22: 5809-13.

34. Uddin G, Rauf A, Collina S, et al. Pistagremic acid, a glucosidase inhibitor from Pistacia integerrima. Fitoterapia 2012;83:1648-52. 